The Physical Signs.

The Condition of the Liver.-A neoplasm is usually associated with a much enlarged and irregular shaped liver, but jaundice may be late in its onset and even not apparent throughout the illness. Syphilis may produce an irregular enlargement which is not so great as that caused by the presence of a growth, and jaundice is not infrequently absent. It has been commonly stated that the liver is diminished in size in alcoholic cirrhosis, and though this is true in a certain proportion of late cases, the majority exhibit a distinct enlargement, and it is then either fairly soft, owing to chronic congestion together with an early cirrhosis without splenic enlargement, or it is hard and even hobnailed and associated with a firm big spleen. A sudden increase in the consumption of alcohol will cause also a sudden increase in size of an already shrunken fibrosed liver which will diminish again under treatment. It may be a most difficult matter to diagnose between an ordinary cirrhosis and a " cirrhosis carcinomatosis," the latter being a diffuse carcinoma spreading throughout a cirrhotic liver. An interesting fact is that 25 per cent. of cases of primary carcinoma of the liver occur in alcoholic cirrhosis, but that such livers are rarely the seat of secondary growths. Diminution in the size of the liver is a feature of acute and subacute yellow atrophy, but here the jaundice is marked, whereas it is a late clinical sign of portal cirrhosis.

Ascites is in favour of a diagnosis of new growth. When met with in alcoholic cirrhosis it is regarded as a sign of advanced disease or the result of concomitant peritonitis. Both ascites and jaundice are induced when the portal vein and the common bile duct become compressed at the hilus of the liver from local enlargement of the lymphatic glands. A similar occlusion, though rare, has been found in a significant number of post-mortem examinations; it was due to aneurysm of the hepatic artery, resulting from a septic embolus in cases of ulcerative endocarditis. A combination of ascites and slight jaundice is observed in cases of heart failure with hepatic congestion, the cyanosis and icterus producing together an almost typical duskygreen colour.

The Gall Bladder and Pancreas.-When the gall bladder is enlarged it is due to some obstruction of the cystic duct or common bile duct, or to a growth of the gall bladder itself. A cystic duct obstruction does not per se lead to jaundice. Courvoisier's famous law is of great value provided that the two fallacies associated therewith are recalled. First, that the gall bladder would not enlarge if previously contracted by the presence of calculi, in spite of a cancerous pancreas; and secondly, that the gall bladder may not suffer fibrosis although stones become impacted in the common duct. The commonest growth producing enlargement of the gall bladder is carcinoma of the head of the pancreas. Radiographic evidence is of value in the detection of gall stones containing calcium salts. In cholecystography we now possess a fresh method in diagnosis; it demonstrates anatomico-pathological changes in the gall bladder, including functional activity, while gall stones that cast no shadows on the radiogram may be shown up as negative shadows on the cholecystogram.

\section{Pathological Examinations.}

of the pathological examinations necessary to confirm a tentative diagnosis the most important is that of the blood. In addition to the van den Bergh test, the Wassermann reaction, and the fragility test of the red cells, there is the blood picture of pernicious anaemia to be romembered, that of Banti's disease with its leucopenia and relative lymphocytosis, and the differential counts in the various infections. Similarly may we diagnose the blood diseases producing jaundice from pressure of enlarged glands.

A high proportion of eosinophile cells indicates a parasitic invasion, especially hydatid, where the diagnosis may be strengthened by carrying out the complement fixation and the precipitin reactions of the patient's blood serum. I have seen one such count in which the eosinophile cells reached the enormous proportion of 70 per cent. of the white cells.
An examination for micro-organisms, or a blood culture, will on occasions be found essential in infections such as malaria, Weil's disease, enteric fever, yellow fever, leptospirosis icterohaemorrhagica, and septicaemic conditions. Weil's disease is now supposed to be due to Bacillus proteus fuorescens, and haemorrhagic jaundice to a leptospira which has been found not only in rat's blood, but also in the mud-slime of mines.

Amoebae or their ova in the stools will give sufficient evidence for a diagnosis of amoebic dysentery with tropical abscess. The chief tests for pancreatitis combine an examination of the stools for an excess of unsplit fat, of the urine for diastase and glucose, and the mydriasis test by adrenaline.

I must refer also to the condition of the bile and other duodenal contents after extraction by means of Einhorn's tube. We can learn therefrom the presence or absence of pus cells, of micro-organisms (such as B. typhosus in carriers), and pancreatic ferments; and would it be saying too much to hope that small roundworms may be the cause of obstruction of the common bile duct? No case of apparent catarrhal jaundice with a tendency to chronicity should be diagnosed as such until, after search for the ova in the stools, or after employment of the therapeutic test, one can conscientiously exclude infestation by Ascaris lumbricoides. An examination of the ascitic fluid reveals its character, whether it be inflammatory, malignant, or transudatory.

Finally I will allude to the tests of hepatic efficiencynumerous and at present somewhat unconvincing, but nevertheless holding a lesser place as an adjuvant in diagnosis.

\section{THE CARDIO-THORACIC INDEX: A CLINICAL METHOD OF EXPRESSING THE SIZE OF THE HEART.* BY}

E. F. SKINNER, M.A., M.B., F.R.C.P., ASSISTANT PHYSICIAN, SHÉFFIELD ROYAL HOSPITAL.

The size of the heart is always of great importance, not only in the examination of individuals suffering from cardiac disease, but also in healthy people when giving an opinion for insurance companies or in compensation claims, and the position of the cardiac impulse, or, as it is rather loosely called, the "apex beat," is generally taken as an indication of the heart's size.

The cardiac impulse is a most valuable physical sign of the heart's action, and its position gives reliable evidence of any change in the situation of the left ventricle, whether this is due to displacement of the whole heart or to hypertrophy and enlargement of this chamber; but it cannot give direct evidence of any cardiac enlargement as a whole.

Accurate determination of the actual size of the heart in any individual is a somewhat lengthy and laborious process, and necessitates very careful - percussion combined with $a$-ray photography; it can hardly be looked upon as a clinical method, but sufficiently accurate information can be obtained by the method to be described.

The accepted definition of the cardiac impulse is the maximum thrust of the left ventricle against the anterior chest wall; this is usually stated to be three and a half to four inches from the mid-sternal line, or, alternatively, half an inch to three-quarters inside the nipple line.

It is quite obvious that the first of these statements can only refer to a certain group of individuals, since the cardiac impulse in a baby of 12 months will bear quite a different relation to the mid-sternal line from that of a child of 12 years, which, again, will be different from that of a man of 50 .

The second statoment, which uses the nipple line as a standard, is even more fallacious, since the nipple varies s. much in position at different ages under different conditions that it is really only of use in dealing with adult men.

* Read to the Sheffield Medico-Chirurgical Society, March, 1926. 
Some simple way of recording the size of the heart would be advantageous, and I have attiempted to find such a method. Some years ago it occurred to me that it might be possible to express the size of the heart as a fraction which would be a constant factor for all ages if the distance of the cardiac impulse from mid-sternum were put as the numerator of a fraction the denominator of which was the circumference of the chest at the level of the impulse. I made a number of measurements, but it was soon obvious that this factor was only a statement of the distance of the cardiac impulse from the middle line. It then appeared possible that an estimation of the deep cardiac dullness expressed as a linear distance from the extreme limit of the right border of the heart to the cardiac impulse, and used as the numeratcr of the fraction, with the circumference of the chest used as the denominator, might give a constant for all ages. But before any conclusions could be reached, it was essential to be sure that the right lorcler of the heart could be determined with sufficient accuracy by clinical percussion to be used in such a measurement. The necessity for this step is evidenced by the frequently expressed opinion that percussion of the right cardiac border is largely a matter of imagination. If due care is observed it is possible to define the right border of the heart with a considerable degree of accuracy, as may be seen in the accompanying drawings (Fig. 1).

The method adopted was to percuss out the borders of the heart with closed eyes and to mark the surface of the chest
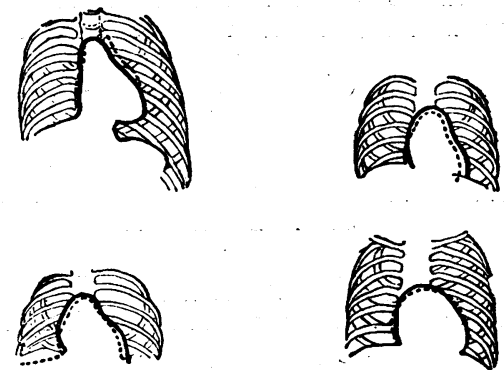

FIG. 1.-Delineation of the cardiac margin.

with a skin pencil; when the percussion was completed, a thin lead wire was run round the pencil marks and kept in position by strips of strapping. An $x$-ray photograph was then taken with the tube carefully focused and centred at a sufficient distance to give parallel rays; the shadow produced by the heart can then be compared with the much denser shadow of the lead wire. I think it will be seen from Fig. 1 that it is possible to delineate the heart's borders with a fair degree of accuracy. Having decided that clinical percussion if carefully and honestly carried out could be relied upon, a number of measurements have been recorded. It appears fairly clear that by expressing the linear cardiac dullness as the numerator and the circumference of the chest as the denominator a fraction is obtained which at any age or sex is almost a constant; it approximates to $1 / 6$, and for the sake of reference $I$ have called this the "cardio-thoracic index." A more accurate numerator would be the distance of the right border of the heart to the impulse, measured by callipers rather than a tape; but the latter is generally available, and the error is a very slight one.

The actual indices obtained by the above method are seen in the accompanying tables, the first of which represents measurements of what may be considered normal conditions. In both tables only the denominator of the fraction is given, for the sake of simplicity. Hers it will be noted that there is only one group in which the fraction is in any way strikingly below $1 / 6$, and this in women between 20 and 40 years of age. It is due to the development of the breasts in this period of life; but even including these low fractions the average for any age of either sex is $1 / 6.3$.

Table II shows a few measurements of abnormal conditions, and I need not detail them, as they are depicted here with the accompanying pathological condition.
TAbLE I.-Normal Cardio-Thoracic Indices.

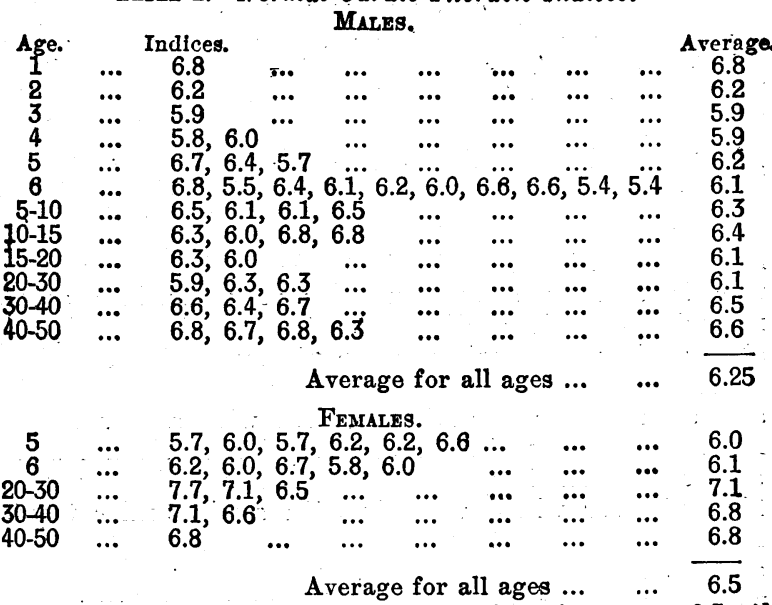

The average index for all ages irrespective of sex was 6.3 ; the lowest variant was 5.4 , the highest variant 7.7.

$$
\begin{aligned}
& \text { Table II.-Abnormal Cardio-Thoracic Indices. } \\
& \text { Age. ... Mitral incompetence; chronic rheumatism... } 5.0 \\
& \text { "... Mitral incompetence, sequel to pneumonia... } 7.0
\end{aligned}
$$

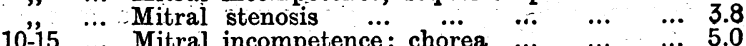

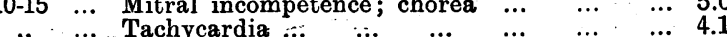

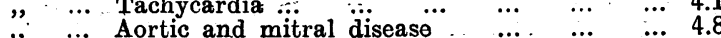

$$
\begin{aligned}
& \text { 15-20 … No cardiac signs; chronic rheumatism; } \\
& \begin{array}{llllllll}
? \text { infantilism } & \ldots & \ldots & \ldots & \ldots & \ldots & 7.2
\end{array}
\end{aligned}
$$

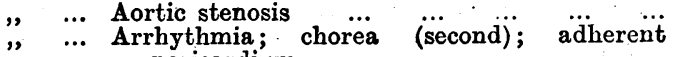

$$
\begin{aligned}
& \text { "... Arrhythmia; } \begin{array}{c}
\text { pericardium } \\
\text {... }
\end{array}
\end{aligned}
$$

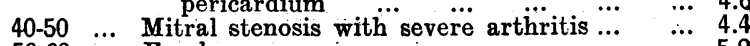

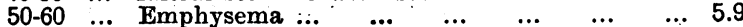

Figs. 2 and 3 are drawings from photographs of actual cyrtometer tracings of the chests of a certain number of cases with the cardiac dullness, circumference, and the

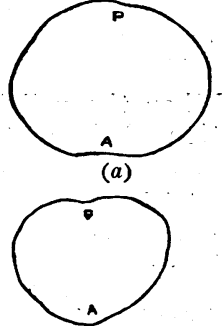

(d)

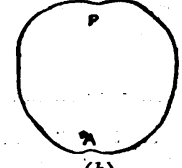

(b)

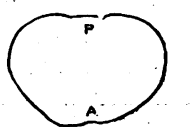

(e)

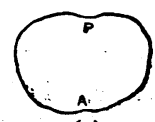

(c)

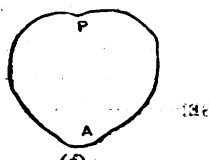

(f)
Fig. 2.-Normal chests. (a) Aged 30, A.P. dia. 8 8 in.; C. dull. $5 \frac{1}{2}$ in. 5 in.: circum. 30 in. C.T.I. $1 / 6$ (c) $($ A A.P. dia. 81 in.; C. dull. 5. dull. 4 in.; circum. 24 in.; C.T.I. $1 / 6$. (d) A.P. dia. 7 in.; C. dull; 4 in.; circum. $26 \frac{3}{4}$ in.; C.T.I. $1 / 6.3$ (e) Aged 13. A.P. dia. $5 \frac{3}{4}$ in.; C. dull. 4 in.; circum. 25 in.; C.T.I. $1 / 6.5$. $(f)$ Aged 22 A.P. dia 73 in.; C. duil. 5 in.; circum. 29 in.; C.T.I. $1 / 5.9$.

$\begin{array}{ll}\text { A.P. dia.= antero-posterior diameter. } & \text { C. dull.=cardiac dullness. } \\ \text { Circum.=circumference of chest. } & \text { C.T.I.=cardio-thoracic index. }\end{array}$

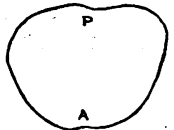

(a)

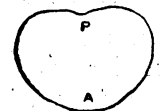

(d)

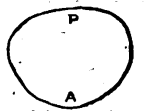

(b)



(e)

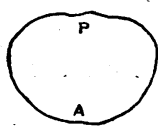

(c)

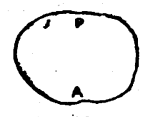

$(f)$

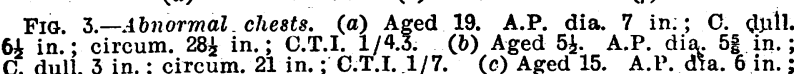
C. duli. 3 in.; circum. 21 in.; C.T.I. 1/7. (c) Aged 15. A.P. dia.6 in.; 5 in.; C. dull. $4 \frac{1}{2}$ in.; circum. 22 in.; C.T.I. 1/4.8. $(e)$ Aged 8. A.P. dia. 5 in ; C. dull. 4 in.; circum. 20 in.; C.T.I. $1 / 5$ (f) Aged 9: January 14th, 1925 : A.P. dia. 5in.; C. dull. 4t in.; circum. 20 in.; circum. 22 in. ; C.T.I. $1 / 3.8$.

cardio-thoracic index shown beside each tracing, together with the age and sex.

Fig. 2 may be considered to represent normal chests, and it will be scen that with wuch different sizes and shapes 
at various ages the actual fraction is remarkably constant. Fig. 3 is that of similar cyrtometer tracings of individuals with abnormal conditions; whilst Fig. 4 shows drawings of what may be called "freak" chests. I merely include

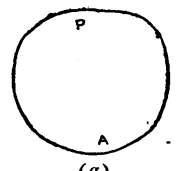

(a)



(c)

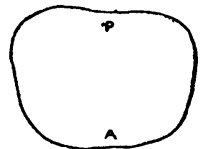

(b)

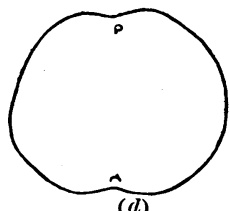

(d)
Fig, 4.-Very abnormal chest8. (a) Aged 13. A.P. dia. 8 in.; C. dull.

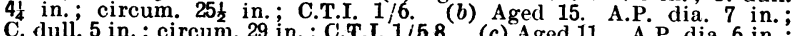
C. duli. 6 in.; circum. 24 ; in. 9 in.; circum. 35 in.

these last by way of interest, and as examples of the one or two conditions in which it is clinically impossible to determine the factor $I$ have called the cardio-thoracic index which is emphasized in Fig. 5, which is an illustration of a

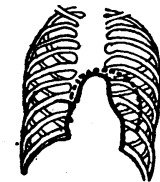

Fig. 5.-The condition in extreme emphysema. chest with extreme emphysema. In such cases, or with chests which are very abnormally shaped, the most careful percussion of which I am capable has failed to map out the deep cardiac dullness. But such cases are comparatively rare, and in them cvery other clinical method is equally fallacious, so they do not materially invalidate the suggestion $I$ have made above-namely, that the size of the heart can be expressed as a constant for any age, the deep cardiac dullness being one-sixth of the circumference of the chest at the level of the cardiac impulse.

\section{ARTIFICIAL PNEUMOTHORAX IN ADVANCED PULMONARY TUBERCULOSIS. BY \\ DENNISON PICKERING, M.D.Cantab., SENIOR PHYSICIAN, PENDYFTRYN HALL SANATORIUM, PENMAENMAWR.}

In the British Medical Journal for February 19th, 1927 (p. 323), a case of pulmonary tuberculosis is described by Dr. Bernard Hudson in which, in spite of a history of old-standing disease with a recent severe "flare-up," an artificial pneumothorax was induced and resulted in considerable and continued improvement. The two following cases are of interest in this connexion.

Casr I.

An ex-officer, aged 26, was admitted to this sanatorium on August 30th, 1922, with a history of persistent cough and cold since the previous winter. Both upper lobes and the upper parts of both lower lobes were involved. The patient improved at first, increasing $28 \mathrm{lb}$. in weight. For the next year he held his own, but then lost ground slowly.

On August 27th, 1925, intermittent haemoptyses began, and on August 29th an artificial pneumothorax was induced. Intermittent bleeding continued for twelve hours, after which there was another haemorrhage of 10 ounces. A refill was given, and the bleeding gradually stopped.

The records of the first operations are as follows :

August, 29th, 1925, 1 a.m. ... $\quad-4,-2 \quad \ldots \quad 1,000$ c.cm. $\quad \ldots \quad-2,+3$

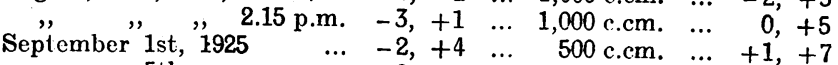
" $\quad 5 \mathrm{th}, \quad, \quad \ldots \quad-2,+5 \quad \ldots \quad 400$ c.cm. $\quad \ldots \quad+2,+6$

The patient gradually recovered from the effects of the haemorrhage and improved in general condition. Fairly high pressures were kept up throughout, and at the beginning of August, 1926, when I took charge of the sanatorium, the last records had been :
July 13th, 1926
$-8,+2$
$800 \mathrm{c.cm}$
$-3,+5$

July $27 \mathrm{th}, 1926 \quad \ldots \quad-10,+2 \quad \ldots .800$ c.cm. $\ldots . \quad+2,+7$

$X$-ray records had not been kept.
When I first saw him the patient had been in bed for four days because his temperature had risen to $102^{\circ}$ for a couple of days after the last refill (June 27th), and he had had vague pains in the left side of the chest. On August 13th I attempted a refill, but found the pressure $-1,+2$. After introducing $150 \mathrm{c.cm}$. of air the pressure was $+2,+3$. On introducing the needle in a different place the pressure was again $+2,+3$, so the attempt a different place the pressure was again $+2,+3$, so the attempt
was abandoned. After this the temperature rose to $99.4^{\circ}$, and was abandoned. After this the temperature rose to $99.4^{\circ}$, and
there were again vague pains in the left side of the chest. About ten days later fluid appeared at the left base, and increased slowly until November $22 n d$, when it had reached the level of the third intercostal space. This was confirmed by $x$-ray examination. Fluid replacement was considered, but in the absence of marked dyspnoea or other signs of cardiac embarrassment, and in view oradually decreased and finally dicted to wait. The fluid much-thickened pleura. The weight, which for a long time had much-thickened pleura. The weight, which for a long time had
remained about 10 st., is now over 10 st. $5 \mathrm{lb}$, temperature and remained about 10 st., is now over 10 st. $5 \mathrm{lb}$., temperature and
pulse are normal, and the patient is walking from three to four pulse are normal, and the patient is walking from three to four found only once since December 30th, 1926. The adventitious signs in the chest have remained about the same, except in so far as they have been modified by the presence of fluid and air respectively.

It is clear that in this case the ultimate prognosis is poor and the immediate risks not inconsiderable. At the same time the pneumothorax, which but for the haemorrhage would never have been attempted, has undoubtedly improved both the chances and the comfort of life. The fluid, by keeping up partial collapse for some months after the refills were stopped, doubtless contributed towards the result.

\section{Case II.}

A girl, aged 20, was admitted in March, 1925, with a history of slight haemoptysis three and a half years previously and recurrence in 1923. On admission, the right upper lobe and the upper half of the lower lobe were involved, and there was some suspicion about the left mid-scapular region. After an $x$-ray examination, Dr. A. E. Barclay of Manchester reported " - . evidence of extensive disease on the right side, which solidated and fibroid the trachea being dragged argely fully three-quarters of an inch to this side... From the interlobar septum downwards there are extensive changes of fibroid type. ... Septum downwards there are extensive changes of fibroid type. On $_{\text {. }}$. which are sharply defined, and do not give the impression of active disease."

In October an unsuccessful attempt to induce an artificia pneumothorax was made. The patient's condition meanwhile remained very much the same.

When I first saw her, in August, 1926, the symptoms were : troublesome cough frequently leading to vomiting, copious sputum swarming with tubercle bacilli, dyspnoea on exertion, and lack of energy. There were signs of definite activity at the left apex of energy. There were signs of definite activity at the left apex,
as well as on the right side. The $x$-ray appearances were much as well as on the right side.

The patient asked me if nothing further could be done. The case did not appear to be particularly suitable for the use of sanocrysin and so, before considering such a line of treatment, or further surgical measures, I felt that another attempt to induce an artificial pneumothorax should be made. The initial operation was performed on September 2nd, 1926, and I was fortunate enough to get into the pleural cavity without difficulty. The earliest to get into the pleural
records are as follows:

$$
\begin{aligned}
& \text { September 2nd, } 1926 \ldots \quad-3,-2 \quad \ldots \quad 250 \text { c.cm. ... } \quad-2,-1
\end{aligned}
$$

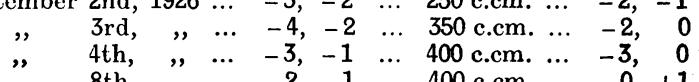

At first only partial collapse was obtained. Almost the whole of the apex was adherent, and there were a number of adhesions near the base. Soon, however, the latter adhesions began to give way gradually, and at the beginning of January collapse was almost complete; the inner parts of the apex and upper lobe, however, remained adherent.

At the beginning of October no râles could be heard on the left side. $X$ rays showed the-trachea in normal position. On November 17th no tubercle bacilli were found, and none have been found since; soon after that date the cough disappeared and the sputum ceased.

There is still some dyspnoea, and a very quiet régime is being There is still some dyspnoea, and a very quiet regime is being followed, with one short walk daily. It has not been possible even less, as re-expansion usually takes place rather rapidly.

In this case, owing to the length of history and the probability of adhesions, the chances of inducing an artificial pneumothorax appeared small. The operation was undertaken rather as a forlorn hope before going on to more serious surgical measures. Yet not only did the operation succeed, but, although the ultimate outlook is doubtful, the chances of life are certainly improved, and in the meantime the absence of severe cough with copious, offensive sputum, and the knowledge that she is no longer a source of danger to others, are in themselves a great boon to the patient. 